\title{
Detecção de Patologias Oculares em Imagens de Reflexo Vermelho Utilizando Descritores de Cor
}

\author{
Caio Manfredini da Silva Martins ${ }^{1}$, Robert Douglas de Araujo Santos ${ }^{1}$, \\ João Dallyson Sousa de Almeida ${ }^{1}$, Geraldo Braz Junior ${ }^{1}$, \\ Jorge Antônio Meireles Teixeira ${ }^{2}$
}

\author{
${ }^{1}$ Núcleo de Computação Aplicada - Universidade Federal do Maranhão (UFMA) \\ São Luís - MA - Brasil \\ ${ }^{2}$ Departamento de Medicina I. - Universidade Federal do Maranhão (UFMA) \\ São Luís - MA - Brasil \\ \{caiomanfredini, robert, jdallyson, geraldo\}enca.ufma.br, \\ jorgemeireleslegmail.com
}

\begin{abstract}
The Brückner Test is an eye exam that aims to detect eye pathologies early. It operates by identifying a red reflection in the eye region after the incidence of a light beam. This work presents a methodology for identifying a possible eye problem in red reflex images from the Brückner test through color descriptors. Using an optimization in the choice of pre-processing methods in conjunction with the dominant color descriptors and color moments, the proposed method reached 92\% accuracy, 98\% specificity, and 76\% sensitivity using the XGBoost classifier.
\end{abstract}

Resumo. O Teste de Brückner é um exame oftalmológico que visa detectar patologias oculares precocemente. Ele opera através da identificação de um reflexo vermelho na região ocular após a incidência de um feixe luminoso. Este trabalho apresenta um novo método para identificação automática de um possível problema ocular em imagens de reflexo vermelho oriundas do Teste de Brückner através de descritores de cor. Utilizando uma otimização na escolha dos métodos de pré processamento em conjunto com os descritores de cor dominante e os momentos de cor, o método proposto alcançou $92 \%$ de acurácia, $98 \%$ de especificidade e $76 \%$ de sensibilidade utilizando o classificador XGBoost.

\section{Introdução}

De acordo com o levantamento realizado pela Organização Mundial de Saúde (OMS), na década de 90 existiam cerca de 38 milhões de pessoas cegas e 110 milhões com baixa acuidade visual. Naquela década, aproximadamente 7 milhões de pessoas perdiam a visão anualmente sendo que esse valor sofria um aumento de entre 1 e 2 milhões a cada ano [Organization et al. 2000]. Além disso, o mesmo levantamento chegou a conclusão que pelo menos dois terços dos casos de cegueira são evitáveis ou tratáveis. Considerando esse cenário, a OMS e a Agência para prevenção de Cegueira (IAPB) lançaram um plano conjunto intitulado de "VISÃO 2020: O direito a Visão". Essa iniciativa pretendia eliminar a cegueira evitável ou tratável até o ano de 2020 adotando como estratégia a assistência oftalmológica básica que por sua vez integrava três fatores: o desenvolvimento de infraestrutura e tecnologias, o controle específico de doenças e o desenvolvimento de recursos 
humanos. No entanto, alguns fatores dificultaram que o plano alcançasse esse objetivo. Dentre eles, pode-se destacar a relação entre a formação de novos oftalmologistas e o aumento da população, que em 2009 era em torno de 6.5 bilhões e que até 2050 estima-se alcançar 9 bilhões de pessoas [Boaretto 2009].

Considerando o panorama no qual a quantidade de médicos oftalmologistas não é capaz de atender a alta demanda populacional [Resnikoff et al. 2012], percebe-se a importância do desenvolvimento de métodos computacionais que possam permitir a detecção de patologias oculares. Tais métodos podem ser utilizados para fornecer acesso básico a saúde da visão para uma maior parcela da população, auxiliar em processos de triagem, servir de segunda opinião para médicos e até mesmo aliviar a carga de trabalho dos profissionais.

Dentre as doenças que causam problemas de visão e até mesmo cegueira estão a miopia, o astigmatismo, a leucoria e o estrabismo. A miopia e o astigmatismo não costumam ser considerados distúrbios graves pois são doenças que podem ser corrigidas através da utilização de óculos com grau, lentes e cirurgias refrativas. Entretanto, existe um alto índice de ocorrências de modo que a OMS reconheceu a miopia como uma das principais causas de deficiência visual [Resnikoff et al. 2008] sendo o público infantil um dos mais afetados [Pan et al. 2012]. Outra doença frequente é a leucoria, que é a existência de um câncer intraocular nomeado retinoblastoma. Apesar de ser majoritariamente diagnosticado ainda na infância, evidências sugerem que muitos portadores são diagnosticados tardiamente [Group 2020]. Por fim, o estrabismo é uma patologia que afeta de $1 \%$ a $4 \%$ da população [Drucker et al. 1997] em pessoas de diversas faixa etárias. Tanto a leucoria quanto o estrabismo podem levar a cegueira caso não sejam tratados precocemente.

Observando que existe uma gama de patologias que afetam a visão podendo causar deficiências graves e até mesmo a cegueira, o Teste de Brückner, também conhecido como Teste do Olhinho, é um exame oftalmológico simples que pode ser empregado para a detecção precoce dessas doenças. O Teste de Brückner consiste na identificação do reflexo vermelho nos olhos do paciente através da incidência de um feixe de luz na região ocular (Figura 1). A ausência de reflexo vermelho visível pode significar a presença de possíveis patologias [Sun et al. 2016] (Figura 1). O recomendado é a realização do exame em todos os bebês entre 1 e 10 meses de vida, visto que as estruturas oculares essenciais para um desenvolvimento visual saudável de uma criança estão em processo de consolidação [Archer 1988]. Além disso, o diagnóstico precoce aumentam as chances de reabilitação do paciente e retardamento de uma possível agravação no quadro.

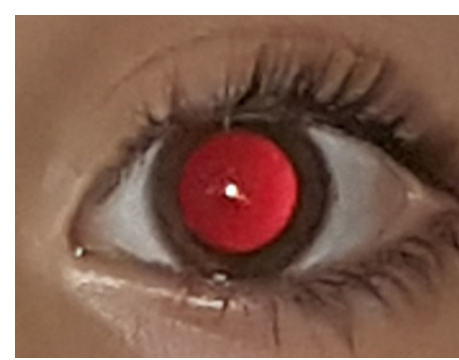

(a) Olho saudável

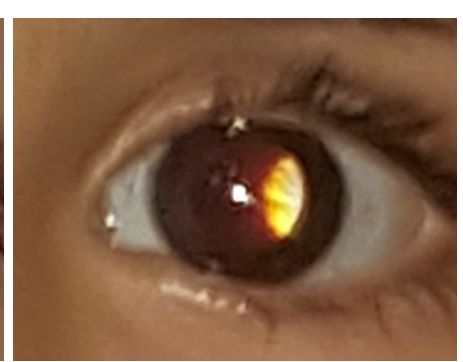

(b) Olho doente

Figura 1. Exemplos de imagens do exame do reflexo vermelho. 
Dando importância às vantagens da detecção precoce de doenças da visão, o presente trabalho propõe um novo método automático, baseado em análise de imagens do reflexo vermelho, desenvolvido para detectar patologias oculares. Para tanto, utiliza descritor de cor dominante [Manjunath et al. 2001] e momentos de cor [Stricker and Orengo 1995] na extração de características em imagens de reflexo vermelho e XGBoost [Chen et al. 2015] para construir um modelo de classificação de olhos saudáveis ou doentes.

\section{Trabalhos Relacionados}

$\mathrm{Na}$ literatura, existem poucos trabalhos que propõem métodos computacionais para classificação de imagens de reflexo vermelho [Silva et al. 2018a] [Rivas et al. 2014]. Silva et al. (2018) utilizaram o classificador REPTree para classificar as características obtidas pelos descritores de Haralick em cada imagem, extraindo um total de 39 características em imagens do mesma base utilizado no nosso trabalho. Rivas et al. (2014) propuseram um método para detecção de leucoria (um tipo patologia ocular caracterizado pelo reflexo branco no Teste de Brückner) utilizando uma arquitetura para classificação nomeada Soft Fusion, a qual utiliza classificadores em conjunto em vez de apenas um. Na extração de caraterísticas, fizeram uso da Transformada Discreta de Cossenos (DCT) e a Transformada de Karhunen-Loève (KLT). Este último trabalho objetivou detectar apenas leucocoria, divergindo tanto no objetivo de classificação quanto na base de imagens.

Apesar da detecção de patologias oculares nos métodos propostos, tais trabalhos não utilizam predominantemente descritores de cor para a eventual classificação. Características de cor são utilizadas amplamente em sistemas de recuperação de imagens [Saluka and Selvarajah 2010] [Mandloi 2014] [Hui Yu et al. 2002]. Em Saluka et al. (2010), a utilização de um conjunto de descritores de cor para a representação de uma imagem obteve bons resultados em comparação a utilização de apenas um. Isso ocorre pelo fato das características extraídas por cada descritor possuírem divergências entre si. Dessa forma, quando utilizados em conjunto, os extratores de características possuem uma maior abrangência na representação de uma imagem.

Dois descritores robustos que obtiveram resultados promissores quanto a classificação de imagens são o descritor de cor dominante [Manjunath et al. 2001] e os momentos de cor [Stricker and Orengo 1995]. O método proposto utiliza tais descritores combinados com a otimização dos métodos de pré-processamento das imagens. A otimização é realizada por meio da escolha das melhores técnicas a serem utilizadas de acordo com a acurácia retornada pela classificação final. Tal método foi utilizado em problemas semelhantes e obteve resultados promissores [Fernandes et al. 2019].

\section{Materiais e método}

O método proposto neste trabalho, ilustrado na Figura 2, consiste em utilizar algoritmos de aprendizado de máquina para realizar a classificação de imagens de reflexo vermelho em normais ou problemáticos. Devido a quantidade de técnicas selecionadas para a realização dos experimentos, optou-se pela utilização de uma otimização para a escolha das melhores. Essa otimização foi realizada através da biblioteca Hyperopt, que utiliza o algoritmo Tree-Structured Parzen Estimator (TPE) [Bergstra et al. 2013] no processo de otimização dos melhores parâmetros. As seções seguintes descrevem cada etapa do método proposto. 


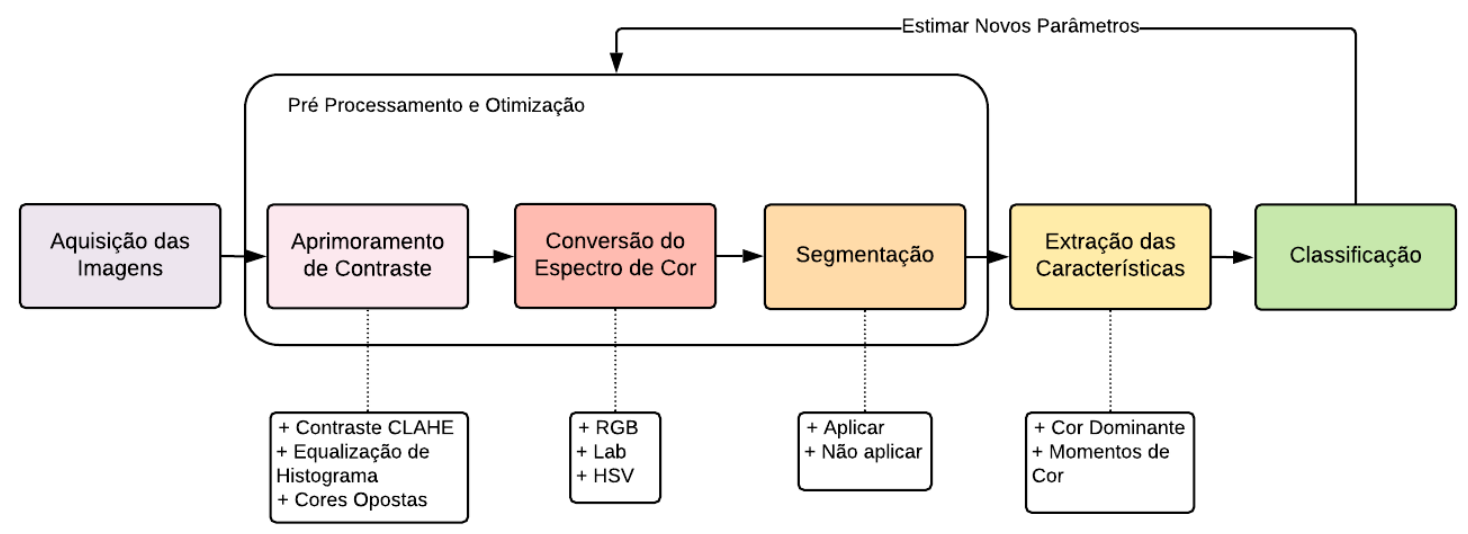

Figura 2. Etapas do método proposto.

\subsection{Aquisição das Imagens}

As imagens utilizadas no presente trabalho foram adquiridas de pacientes em uma clínica oftalmológica em São Luís-MA, por [Silva et al. 2018a] utilizando uma câmera com a função flash ativada. Os pacientes que se voluntariaram a participar do experimento assinaram um formulário de consentimento. Esse estudo recebeu a aprovação do comitê de ética para o uso de imagens humanas.

Durante a aquisição das imagens foi solicitado que pacientes estivessem com as pupilas dilatadas e não utilizassem óculos, pois este é o procedimento padrão do Teste de Brückner. A base contém 58 fotografias de faces e está dividida em duas classes: pacientes normais (33 imagens) e pacientes com patologia (25 imagens).

\subsection{Pré-Processamento e Otimização}

A etapa de pré-processamento tem como objetivo remover ruídos e trazer representações mais assertivas das imagens carregadas. Isso pode ser realizado através de filtros e transformações que são aplicados em cada imagem. O pré-processamento, quando aplicado devidamente, tem um grande impacto na fase de extração de caraterísticas [Gonzalez and Woods 2010]. Dessa forma, a escolha das melhores técnicas de préprocessamento é essencial para o bom desempenho do algoritmo de classificação. $O$ procedimento de aprimoramento de contraste, como parte do pré-processamento, é bastante conhecido no campo da visão computacional. Geralmente é responsável por reduzir ruídos realçar as cores nas imagens. Existem várias técnicas existentes para alcançar esse objetivo, entretanto, os experimentos foram limitados a três métodos: Equalização de Histograma, Equalização adaptativa de histograma com limitação de contraste (CLAHE)[Pizer et al. 1987] e Cores Opostas [Silva et al. 2018b].

Outro procedimento bastante comum às etapas de pré-processamento é a transformação do espaço de cor. Através dessa transformação, é possível obter novas representações de cores das imagens, produzindo características não observáveis no espectro de cor original. Os experimentos foram realizados utilizando três espectros: RGB (red, green, blue), HSV (hue, saturation, value) e Lab (luminosity, green-red, blueyellow). Por fim, o último procedimento selecionado para ser realizado foi a Segmentação 
da região do reflexo vermelho. Dessa forma, as caraterísticas a serem extraídas não sofreriam interferência de possíveis erros relacionados a tonalidade de pele ou cores externas à região de interesse.

A fim de estabelecer a melhor combinação possível dos métodos de préprocessamento, optou-se pela utilização de um otimizador para seleção automática das técnicas selecionadas. O otimizador foi realizado sobre a biblioteca HyperOpt que disponibiliza o algoritmo Tree-Structured Parzen Estimator (TPE) [Bergstra et al. 2013]. Esse algoritmo utiliza decisões bayesianas para escolha de parâmetros, de maneira que a convergência para encontrar a melhor parametrização é mais rápida quando comparada com a decisão aleatória ou usando GridSearch. Nas subseções seguintes contém os métodos disponibilizados em cada subetapa para escolha automática pelo Hyperopt.

\subsubsection{Aprimoramento de Contraste}

O Aprimoramento de Contraste tem um papel significativo na representação visual de imagens para a visão computacional. Geralmente, imagens com baixa luminosidade e contraste podem prejudicar o desempenho da classificação futura. A Equalização de Histograma [Gonzalez and Woods 1992] é uma técnica simples bastante utilizada para realçar as cores de uma imagem [Wong et al. 2016] [Abdullah-Al-Wadud et al. 2007]. Ela opera através da redistribuição dos níveis de intensidade do histograma de cores da imagem, nivelando uniformemente todo os intervalos de cores. Muitas propostas foram implementadas realizando variações da Equalização de Histograma [Abdullah-Al-Wadud et al. 2007] [Veluchamy and Subramani 2019]. Entretanto, optouse pela utilização da implementação original devido ao seu desempenho e bons resultados em problemas semelhantes.

Outra técnica utilizada foi o CLAHE. Este método de realce é amplamente utilizado em problemas oftálmicos para a detecção automática de micro aneurismas ou segmentação de vasos sanguíneos da retina [Kurt et al. 2012]. O CLAHE opera através da equalização adaptativa do histograma que fornece um aumento de contraste baseado em pequenas partições da imagem. O histograma de cores de cada partição é calculado de acordo com o clip máximo previamente definido, resultado em uma imagem com mais brilho, sem deformar características importantes.

Por fim, o contraste com Cores Opostas foi implementado. Define-se uma cor oposta (ou complementar) aquelas que dentro de um círculo cromático são posicionadas na extremidade oposta. Dessa forma, ao aplicar o filtro, as cores de uma imagem são invertidas para suas cores complementares. O objetivo da utilização dessa técnica é trazer uma nova interpretação da imagem e evidenciar o reflexo vermelho. A Figura 3 mostra um exemplo de imagem original da base antes e após aplicação das técnicas de aprimoramento de contraste.

\subsubsection{Definição dos Espectros de Cor}

Em geral, o espectro de cor RGB (red, green, blue) é o mais utilizado em telas com imagens digitais e instrumentos óticos. O padrão de disposição segue um modelo que 


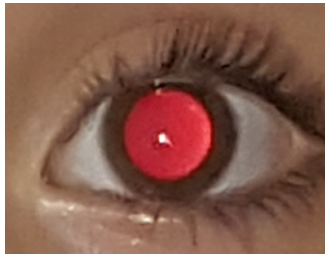

(a) Imagem Original

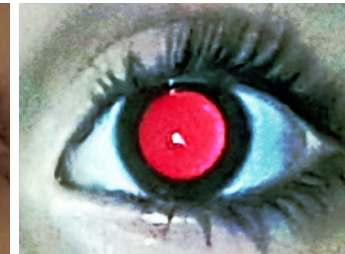

(b) Eq. de Histograma

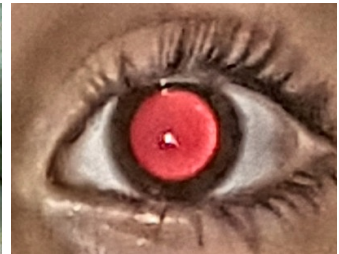

(c) CLAHE

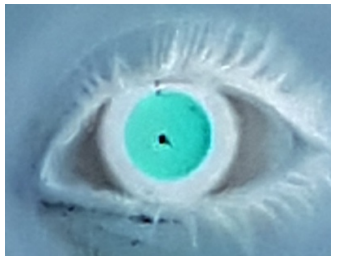

(d) Cores Opostas

Figura 3. Representação dos métodos de aprimoramento.

relaciona três cores principais: vermelho, verde e azul. Dessa forma, todo pixel de uma imagem possui uma correspondência em cada um dos três canais. Por ser o modo de leitura padrão, diversas aplicações utilizam esse espectro de cor original para processar imagens. Entretanto, têm surgido novos métodos que utilizam a transformação de espectro antes da extração das características [Stricker and Orengo 1995, Vass et al. 1998, Desai et al. 2010].

Já o espaço de cor HSV (hue, saturation, value ou matiz, saturação e valor) é uma das transformações que tem sido bastante utilizada na área de classificação de imagens [Junhua and Jing 2012, Indriani et al. 2017]. Hue (ou matiz) é referente a propriedade da cor, como o azul, verde, vermelho etc. Saturation (ou saturação) é a intensidade da cor em específico (vermelho claro, vermelho escuro). Value (valor) é o nível de brilho existente na cor analisada. Esses canais quando combinados trazem maior nível de detalhes quando comparado ao RGB. Além do HSV, outro espaço utilizado em aplicações semelhantes é o Lab (ou CIELAB). Ele representa a cor através de três canais: $L$, que representa a luminosidade, e os canais $a$ e $b$ que representam as quatro cores essenciais na visão humana (vermelho, verde, azul e amarelo).

Cada espectro selecionado possui bons resultados em sistemas classificadores de imagens. Entretanto, possuem divergência entre si.

\subsubsection{Segmentação do reflexo vermelho}

A base disponibilizada possui em sua composição a segmentação da íris nas imagens dos olhos. Dessa forma, não foi realizada a implementação de uma etapa para a segmentação automática do reflexo. Entretanto, em trabalhos futuros, pretende-se adicionar essa etapa à arquitetura. Nos experimentos, a otimização realizado pelo Hyperopt definiu se a segmentação deveria ser aplicada ou não. Ou seja, foi verificado se os melhores resultados são alcançados com a região do reflexo segmentada ou com a imagem da região do olhos, apenas.

\subsection{Extratores de Características}

O processo de extração de características tem como objetivo transformar uma imagem em valores numéricos para uma futura classificação. As características obtidas podem servir para diversos objetivos como encontrar certos objetos na imagem, encontrar padrões em cores e texturas. Existem também diversas informações possíveis que podem ser extraídas da imagem que podem variar de acordo com o problema em questão [Sklansky 1978]. Como exemplo, pode-se citar os descritores de forma, de textura ou de espaço. Entre- 
tanto, devido ao escopo do problema abordado neste trabalho, o poder discriminativo das cores foi considerado o mais essencial no desenvolvimento do método. Dessa forma, foram utilizados majoritariamente descritores de cor para a etapa de extração de características. Os experimentos foram conduzidos utilizando dois descritores: o descritor de cor dominante e os momentos de cor.

O descritor de cor dominante [Manjunath et al. 2001] é bastante utilizado na área da visão computacional em sistemas de recuperação de imagens que são baseados em conteúdo (BIR - content-based image retrieval). Ele opera através do agrupamento (ou clusterização) das cores de uma imagem, retornando uma menor quantidade de cores e suas determinadas porcentagens na imagem (o que passa a representar a imagem como um todo). Dessa forma, o agrupamento opera sobre cada canal da imagem, encontrando um conjunto de centroides e suas respectivas ocorrências (ou aproximações) em cada canal individualmente.

Outro descritor utilizado foram os momentos de cor, que mensuram a distribuição das cores em uma imagem [Stricker and Orengo 1995]. A implementação dos momentos de cor fundamenta-se no pressuposto de que a distribuição das cores em uma imagem podem ser traduzidas como uma distribuição de probabilidades. Se as cores de uma imagem seguem uma certa distribuição de probabilidade, os momentos dessa distribuição podem ser usados como características para identificar outras imagens com distribuições semelhantes.

Os momentos de cor podem ser definidos como três valores que são calculados em uma imagem. O primeiro momento de cor é a Média. O segundo momento de cor é o Desvio Padrão. Por fim, o terceiro momento de cor é a Distorção. Os três valores são extraídos de cada canal de uma imagem, resultando em nove características finais.

Definindo $N$ como o número de pixels da imagem e $p_{i j}$ sendo o valor do pixel $j$ da imagem no canal $i$, o cálculo da Média (primeiro momento de cor) é realizado através da Equação 1:

$$
E_{i}=\sum_{j=1}^{N} \frac{1}{N} p_{i j}
$$

Definindo $E_{i}$ como o valor da Média (primeiro momento de cor), o Desvio Padrão (segundo momento de cor) é definido através da Equação 2:

$$
\delta_{i}=\sqrt{\left(\frac{1}{N} \sum_{j=1}^{N}\left(p_{i j}-E_{i}\right)^{2}\right)}
$$

3:

O terceiro momento de cor denominado Distorção é calculado através da Equação

$$
\gamma_{i}=\sqrt[3]{\left(\frac{1}{N} \sum_{j=1}^{N}\left(p_{i j}-E_{i}\right)^{3}\right)}
$$


É importante ressaltar que as características obtidas pelos descritores implementados são invariantes a atributos espaciais. Isso significa que aspectos espaciais (como a localização de um fenômeno) não podem ser mensurados. Os resultados obtidos dos experimentos realizados são expostos nos tópicos seguintes.

\subsection{Classificação}

Os classificadores utilizados foram o SVM, Random Forest e o XGBoost. O treinamento e o teste das instâncias seguiram o método de validação cruzada com 10 folds. Os extratores de características utilizados foram os descritores de cor dominante concatenados com os momentos de cor.

\section{Resultados e Discussão}

Os experimentos foram realizados em dois fluxos distintos. No primeiro fluxo, os testes foram realizados sem a utilização da otimização no pré-processamento, porém, aplicando a segmentação da região de interesse. Esse passo foi realizado para verificar se a otimização influência na classificação inicial dos algoritmos. Os resultados da execução dessa etapa podem ser visualizados na Tabela 1, organizados por classificador, acurácia (Acu.), especificidade (Esp.) e sensibilidade (Sen.). Estão ordenados de maneira descendente a partir da acurácia.

\begin{tabular}{||cccc||}
\hline Classificador & Acu. & Esp. & Sen. \\
\hline \hline XGBoost & $89 \%$ & $96 \%$ & $78 \%$ \\
Random Forest & $86 \%$ & $96 \%$ & $69 \%$ \\
SVM & $80 \%$ & $98 \%$ & $44 \%$ \\
\hline
\end{tabular}

Tabela 1. Resultados primários (sem otimização).

No segundo fluxo, os testes foram realizados utilizando a otimização dos métodos de pré processamento. Na etapa de Aprimoramento de Contraste foram utilizados seguinte parâmetros: Equalização de Histograma, Contraste CLAHE e Cores Opostas. Na etapa de transformação do espectro de cor os parâmetros possíveis foram: RGB, HSV e Lab. Na etapa de segmentação, era possível aplicar a segmentação da área do reflexo vermelho ou não. A otimização foi realizada para cada um dos classificadores usados no primeiro fluxo, totalizando cerca de 50 testes. Os quatro melhores resultados ordenados a partir da acurácia podem ser observados na Tabela 2.

\begin{tabular}{||ccccccc||}
\hline Aprimoramento de Contraste & Espaço de Cor & Segmentação & Classificador & Acu. & Esp. & Sen. \\
\hline \hline Equalização Hist. & Lab & Sim & XGBoost & $92 \%$ & $98 \%$ & $76 \%$ \\
CLAHE & Lab & Sim & XGBoost & $91 \%$ & $96 \%$ & $71 \%$ \\
Equalização Hist & RGB & Sim & RandomForest & $88 \%$ & $94 \%$ & $75 \%$ \\
Cores Oppostas & HSV & Sim & XGBoost & $87 \%$ & $94 \%$ & $75 \%$ \\
\hline
\end{tabular}

Tabela 2. Resultados secundários (com otimização).

Após a realização dos testes, algumas suposições podem ser realizadas a respeito da melhor convergência dos métodos testados. É observável que a realização da segmentação é essencial, visto que em todos os melhores resultados ela esteve presente. 
Além disso, o classificador XGBoost foi o mais selecionado nas classificações mais assertivas. Por fim, os métodos de pré-processamento que menos alteram a representação original das cores contribuíram para a melhor classificação final.

As imagens de pacientes portadores do estrabismo foram as que o método mais falhou na classificação. Principalmente, devido as características extraídas não possuírem representação espacial, necessária para identificar a existência de desalinhamento ocular. Dessa forma, pacientes portadores do estrabismo que possuem um reflexo vermelho bastante acentuado nos olhos, não foram classificados corretamente pelo método proposto. A Figura 4 ilustra este cenário.

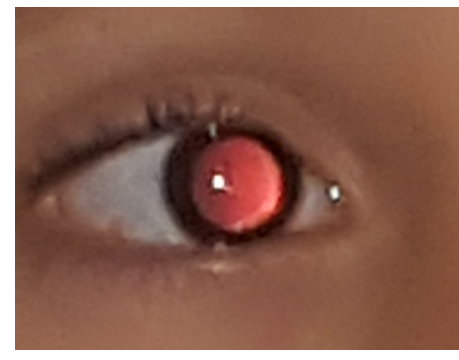

(a)

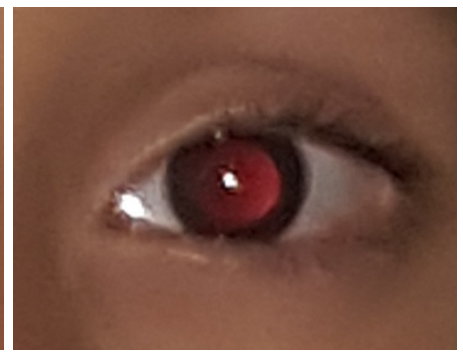

(b)

Figura 4. Exemplo de reflexo vermelho do olhos de um paciente portador de estrabismo.

A Tabela 3 apresenta o comparativo do melhor resultado obtido pelo método proposto comparado aos resultados dos trabalhos de Silva et al. (2018) e Rivas et al. (2014). Dentre os trabalhos citados, Silva et al. (2018) é o mais próximo da nossa abordagem, mantendo a acurácia e a especificidade levemente inferiores ao presente trabalho. Entretanto, obteve sensibilidade mais elevada.

\begin{tabular}{||c|l|c|c|c||}
\hline & Abordagem & Acu. & Esp. & Sen. \\
\hline \hline Silva et al. 2018 & REPTree com descritores Haralick & $91 \%$ & $91 \%$ & $90 \%$ \\
Rivas et al. 2014 & Soft Fusion com DCT e KLT & $88 \%$ & - & - \\
Método proposto & XGBoost com Momentos de Cor e Cores Dominantes & $\mathbf{9 2 \%}$ & $\mathbf{9 8 \%}$ & $\mathbf{7 6 \%}$ \\
\hline
\end{tabular}

Tabela 3. Comparação dos resultados.

\section{Conclusão e Trabalhos Futuros}

A partir dos resultados obtidos, observa-se que o método proposto alcançou resultados promissores. No entanto, para garantir a validação do algoritmo implementado, é necessário avaliá-lo em um maior conjunto de imagens. Para isso, visa-se a realização do Data Augmentation, que estende a base de dados e cria novas imagens a partir das imagens já pré existentes na base.

Além disso, pretende-se realizar a segmentação automática da região do reflexo vermelho para construir uma ferramenta de detecção automática de patologias da visão. A arquitetura de otimização desenvolvida possibilita a adição de novos métodos de préprocessamento, assim como novos métodos de extração de características relacionadas a cor. Portanto, para trabalhos futuros, sugere-se a utilização de novos parâmetros, estendendo a otimização para a etapa de extração de características, incluindo abordagens de extração de características espaciais. 


\section{Referências}

Abdullah-Al-Wadud, M., Kabir, M. H., Akber Dewan, M. A., and Chae, O. (2007). A dynamic histogram equalization for image contrast enhancement. IEEE Transactions on Consumer Electronics, 53(2):593-600.

Archer, S. M. (1988). Developmental aspects of the brückner test. Ophthalmology, 95(8):1098-1101.

Bergstra, J., Yamins, D., and Cox, D. D. (2013). Hyperopt: A python library for optimizing the hyperparameters of machine learning algorithms. In van der Walt, S., Millman, J., and Huff, K., editors, Proceedings of the 12th Python in Science Conference, pages $13-20$.

Boaretto, A. E. (2009). A evolução da população mundial, da oferta de alimentos e das ciências agrárias. Revista Ceres, 56(4):513-526.

Chen, T., He, T., Benesty, M., Khotilovich, V., Tang, Y., Cho, H., et al. (2015). Xgboost: extreme gradient boosting. R package version 0.4-2, 1(4).

Desai, P., Pujari, J., and S.N, P. (2010). Content-based image retrieval using color and shape descriptors. In Arabian Journal for Science and Engineering.

Drucker, H., Burges, C. J., Kaufman, L., Smola, A., Vapnik, V., et al. (1997). Support vector regression machines. Advances in neural information processing systems, 9:155-161.

Fernandes, A. G., Martins, C., Lima, A. C., Junior, G., de Almeida, J. D., and de Paiva, A. (2019). Meta aprendizagem de extração de caracteristicas aplicada ao diagnostico de glaucoma. In Anais do XIX Simpósio Brasileiro de Computação Aplicada à Saúde, pages 342-347, Porto Alegre, RS, Brasil. SBC.

Gonzalez, R. C. and Woods, R. E. (1992). Digital image processing. Reading:AddisonWesley, pages 85-103.

Gonzalez, R. C. and Woods, R. E. (2010). Processamento Digital de Imagens. Pearson, $3^{\mathrm{a}}$ edition.

Group, G. R. S. (2020). Global Retinoblastoma Presentation and Analysis by National Income Level. JAMA Oncology, 6(5):685-695.

Hui Yu, Mingjing Li, Hong-Jiang Zhang, and Jufu Feng (2002). Color texture moments for content-based image retrieval. In Proceedings. International Conference on Image Processing, volume 3, pages 929-932 vol.3.

Indriani, O. R., Kusuma, E. J., Sari, C. A., Rachmawanto, E. H., and Setiadi, D. R. I. M. (2017). Tomatoes classification using k-nn based on glcm and hsv color space. In 2017 International Conference on Innovative and Creative Information Technology (ICITech), pages 1-6.

Junhua, C. and Jing, L. (2012). Research on color image classification based on hsv color space. In 2012 Second International Conference on Instrumentation, Measurement, Computer, Communication and Control, pages 944-947. 
Kurt, B., Nabiyev, V. V., and Turhan, K. (2012). Medical images enhancement by using anisotropic filter and clahe. In 2012 International Symposium on Innovations in Intelligent Systems and Applications, pages 1-4.

Mandloi, G. (2014). A survey on feature extraction techniques for color images. In International Journal of Scientific and Engineering Research.

Manjunath, B., Ohm, J., Vasudevan, V., and Yamada, A. (2001). Color and texture descriptors. Circuits and Systems for Video Technology, IEEE Transactions on, 11:703 715 .

Organization, W. H. et al. (2000). Global initiative for the elimination of avoidable blindness. Technical report, World Health Organization.

Pan, C.-W., Ramamurthy, D., and Saw, S.-M. (2012). Worldwide prevalence and risk factors for myopia. Ophthalmic and Physiological Optics, 32(1):3-16.

Pizer, S. M., Amburn, E. P., Austin, J. D., Cromartie, R., Geselowitz, A., Greer, T., ter Haar Romeny, B., Zimmerman, J. B., and Zuiderveld, K. (1987). Adaptive histogram equalization and its variations. Computer Vision, Graphics, and Image Processing, 39(3):355-368.

Resnikoff, S., Felch, W., Gauthier, T.-M., and Spivey, B. (2012). The number of ophthalmologists in practice and training worldwide: a growing gap despite more than 200 000 practitioners. British Journal of Ophthalmology, 96(6):783-787.

Resnikoff, S., Pascolini, D., Mariotti, S. P., and Pokharel, G. P. (2008). Global magnitude of visual impairment caused by uncorrected refractive errors in 2004. Bulletin of the World Health Organization, 86:63-70.

Rivas, P., Baker, E., Hamerly, G., and Shaw, B. (2014). Detection of leukocoria using a soft fusion of expert classifiers under non-clinical settings. BMC ophthalmology, $14: 110$.

Saluka, K. and Selvarajah, S. (2010). Comparison of color features for image retrieval. Indian Journal of Computer Science and Engineering, 1.

Silva, I. F. S., Almeida, J. D. S., Teixeira, J. A. M., Júnior, G. B., and de Paiva, A. C. (2018a). Teste automático de brückner basedo em imagens. In Anais do XVIII Simpósio Brasileiro de Computação Aplicada à Saúde, Porto Alegre, RS, Brasil. SBC.

Silva, M. G., Pessoa, A. C., de Almeida, J. D., Junior, G. B., and de Paiva, A. C. (2018b). Diagnóstico do glaucoma em imagens de retinografia usando variantes de padroes locais binários. In Anais do XVIII Simpósio Brasileiro de Computação Aplicada à Saúde. SBC.

Sklansky, J. (1978). Image segmentation and feature extraction. IEEE Transactions on Systems, Man, and Cybernetics, 8(4):237-247.

Stricker, M. and Orengo, M. (1995). Similarity of color images. Proceedings of SPIE The International Society for Optical Engineering, 2420.

Sun, M., Ma, A., Li, F., Cheng, K., Zhang, M., Yang, H., Nie, W., and Zhao, B. (2016). Sensitivity and specificity of red reflex test in newborn eye screening. The Journal of pediatrics, 179:192-196. 
Vass, J., Jia Yao, Joshi, A., Palaniappan, K., and Xinhua Zhuang (1998). Interactive image retrieval over the internet. In Proceedings Seventeenth IEEE Symposium on Reliable Distributed Systems (Cat. No.98CB36281), pages 461-466.

Veluchamy, M. and Subramani, B. (2019). Image contrast and color enhancement using adaptive gamma correction and histogram equalization. Optik, 183:329-337.

Wong, C. Y., Jiang, G., Rahman, M. A., Liu, S., Lin, S. C.-F., Kwok, N., Shi, H., Yu, Y.-H., and Wu, T. (2016). Histogram equalization and optimal profile compression based approach for colour image enhancement. Journal of Visual Communication and Image Representation, 38:802-813. 